\title{
Identification of HeLa cell proteins that interact with Chlamydia trachomatis glycogen synthase using yeast two-hybrid assays
}

\author{
ZHENJIE SUN $^{1 *}$, YUHUI SUN ${ }^{1 *}$, YUMENG LI $^{1}$, XIULI LUAN $^{1}$, \\ HUI CHEN ${ }^{1}$, HAIYING WU ${ }^{1}$, BO PENG ${ }^{2}$ and CHUNXUE LU ${ }^{1}$ \\ ${ }^{1}$ Institution of Pathogenic Biology and ${ }^{2}$ Department of Pathology, Hengyang Medical College, \\ University of South China, Hengyang, Hunan 421001, P.R. China
}

Received June 27, 2019; Accepted November 18, 2019

DOI: $10.3892 / \mathrm{mmr} .2020 .10947$

\begin{abstract}
Chlamydia trachomatis (C. trachomatis) is the leading cause of bacterial sexually transmitted diseases and infectious diseases that cause blindness. The pathophysiology of chlamydial infections is poorly understood, but secreted proteins have emerged as key virulence factors. $C$. trachomatis glycogen synthase $(\mathrm{Glg} A)$ is a chlamydial secretory protein, which localizes in the lumen of chlamydial inclusion bodies and the cytosol of host cells. In order to improve understanding of the roles of $\mathrm{Glg} \mathrm{A}$ in chlamydial pathogenesis, four proteins that interact with GlgA, Homo sapiens CXXC finger protein 1, prohibitin (PHB), gelsolin-like actin-capping protein and apolipoprotein A-I binding protein were identified using yeast two-hybrid assays. The functions of these proteins are complex, and preliminary results suggested that PHB interacts with $\mathrm{GlgA}$. However, further studies are required to determine the specific interactions of these proteins with GlgA. The findings of the present study may provide a direction and foundation for future studies focusing on the mechanism of GlgA in C. trachomatis infection.
\end{abstract}

\section{Introduction}

Chlamydia is a genus of gram-negative obligate intracellular bacteria consisting of nine recognized species; each species exhibits specific tissue tropism and disease pathology (1). The global impact of Chlamydia trachomatis (C. trachomatis) is considerable, and of significant medical concern in

Correspondence to: Professor Chunxue Lu, Institution of Pathogenic Biology, Hengyang Medical College, University of South China, 28 Changsheng Road, Zhengxiang, Hengyang, Hunan 421001, P.R. China

E-mail: lcxpbdudu@126.com

${ }^{*}$ Contributed equally

Keywords: Chlamydia trachomatis, glycogen synthase, yeast two-hybrid assay humans (2). C. trachomatis exists as 19 serovars; serovars A-C are responsible for trachoma, the leading infectious cause of blindness worldwide (3). Serovars D-K primarily infect the genital mucosae, causing numerous commonly-diagnosed sexually transmitted diseases, including hydrosalpinx, a laparoscope-detectable marker of tubal factor infertility (4). Moreover, C.trachomatis is also a major risk factor in the transmission of human immunodeficiency virus (5). To the best of our knowledge, no study to date has determined why C. trachomatis can cause infectious blindness, or how infection of the lower genital tract can result in tubal fibrosis and hydrosalpinx. Therefore, the aim of the present study was to determine the molecular mechanisms of $C$. trachomatis pathogenicity, and to guide the design of live-attenuated vaccine strains for the prevention of chlamydial diseases.

As with all other chlamydia, $C$. trachomatis possesses a unique intracellular growth cycle with a distinct biphasic developmental cycle, alternating between an infectious elementary body (EB) and a replicating, metabolically-active reticulate body (RB) (6). EBs differentiate into RBs within a non-acidified vacuole, the chlamydial inclusion (7). At $\sim 18 \mathrm{~h}$ post-infection, the generated progeny differentiate back into EBs. Later in the developmental cycle, EBs are released from the host cell to initiate a new cycle of infection (2).

Host inflammatory responses triggered by chlamydial intracellular survival and replication contribute to chlamydia-induced pathologies; secretory proteins, including chlamydia protease-like activity factor (CPAF), have been hypothesized to play important roles in this process (8). CPAF, secreted into the cytosol of $C$.trachomatis-infected cells, is involved in the immune evasion mechanism that may block $\mathrm{NF}-\kappa \mathrm{B}$ p 65 nuclear translocation, resulting in decreased interferon- $\beta$ and pro-inflammatory cytokine synthesis (9). Despite extensive research into CPAF, the mechanism of C. trachomatis-associated trachoma and infertility following infection remains unknown. Asmost chlamydia-infected host cells secrete chlamydial proteins, other secreted proteins are also likely to notably contribute tothe pathogenicity of chlamydial infections in humans.

In a previous study, in fixed $C$.trachomatis-infected HeLa cells analyzed with antibodies against chlamydia glycogen 
metabolism-related enzymes, only $C$. trachomatis glycogen synthase (GlgA) was found to be secreted into the host cell cytosol (10). It was first revealed to be associated with chlamydial inclusion bodies at $12 \mathrm{~h}$ post-infection, and secretion into the cytosol was detectable at $\sim 24 \mathrm{~h}$ post-infection. However, since glycogen was only monitored in the inclusion bodies, and not the cytosol, it is unclear whether GlgA secretion into the host cell cytosol is necessary for the induction of chlamydial diseases.

GlgA expression is dependent on a cryptic plasmid; removal of this plasmid results in the loss of GlgA expression and attenuated pathogenicity in both C.trachomatis serovar A and C. muridarum (11). These findings indicate that GlgA may play an essential role in chlamydial pathogenesis.

The yeast two-hybrid system enables the in vivo detection of interacting proteins in order to reveal the biological roles of a known protein (12). Following a series of optimizations and development by Fields and Song (13), the yeast two-hybrid system was considered to bea classical method ofidentifying and studying protein-protein interactions. In a recent studyusing the yeast two-hybrid system, the C.trachomatis inclusion membrane protein MrcAwas found tointeract with inositol 1,4,5-trisphosphate receptor type 3 to regulate extrusion formation (14). Thus, due to its inexpensive and time-saving nature, the yeast two-hybrid system is a powerful method for the analysis of protein-protein interactions.

In the present study, the yeast two-hybrid system was used to identify proteins that interact with GlgA. This strategy involved screening 13 potential clones, which following cDNA identification, were confirmed via rotary validation and co-immunoprecipitation. The results indicated that prohibitin (PHB) interacts with GlgA, which may provide novel insight into the understanding of GlgA in chlamydial biology and pathogenesis.

\section{Materials and methods}

Bait plasmid construction. The Matchmaker two-hybrid system (Clontech Laboratories, Inc.) was used to confirm the potential interaction partners of GlgA (CT798). The gene sequence of CT798 (WP-100139618) was obtain from the National Centre for Biotechnology Information database (https://www.ncbi.nlm.nih. gov/) and amplified by PCR using the following primers, which contained SfiI sites at both ends: CT798 forward, 5'-AAGGCC ATTACGGCCATGAAAATTATTCACACAGCTATCG-3' and reverse, 5'-CCGGCCGAGGCGGCCTTGTTTATAAATTTC TAAATATTTATTGGC-3'.

PCR was performed under the following conditions: Initial denaturation at $98^{\circ} \mathrm{C}$ for $5 \mathrm{~min}$, amplification (30 cycles) at $98^{\circ} \mathrm{C}$ for $30 \mathrm{sec}, 55^{\circ} \mathrm{C}$ for $30 \mathrm{sec}$ and $72^{\circ} \mathrm{C}$ for $42 \mathrm{sec}$, and afinal extension at $72^{\circ} \mathrm{C}$ for 5 min (Qiagen). The FastPfu DNA Polymerase was used (Beijing Transfen Biotech Co., Ltd.). The orientation and authenticity of the plasmid sequence was confirmed by sequencing (Sangon Biotech Co., Ltd.) and restriction endonuclease digestion by $8 \%$ SfiI digestion (Fermentas) at $50^{\circ} \mathrm{C}$ for $24 \mathrm{~h}$.

Recombinant plasmid confirmation. The CT798 plasmid was cloned into the GAL4 binding domain of the pGBKT7 vector (Hitech Bio-Technology Co., Ltd). CT798 plasmids were treated with $S f i$ in a $25-\mu 1$ reaction system including $20 \mu \mathrm{l}$ CT798, $2 \mu \mathrm{l} S f i \mathrm{I}$ restriction enzyme, $2.5 \mu \mathrm{l} 10 \mathrm{X}$ buffer and $0.5 \mu 1$ sterile water. pGBKT7 was treated with $S f$ iI in a $20-\mu 1$ reaction system including $10 \mu \mathrm{l}$ CT798, $1 \mu \mathrm{l}$ SfI restriction enzyme, $2 \mu \mathrm{l} 10 \mathrm{X}$ buffer and $7 \mu \mathrm{l}$ sterile water. The digested reaction mixtures were then combined and incubated at $16^{\circ} \mathrm{C}$ for $1 \mathrm{~h}$, consisting of $5 \mu \mathrm{l}$ Ligation High Buffer (Toyobo Life Sciences), $5 \mu \mathrm{l}$ CT798/SfiI and $0.5 \mu 1 \mathrm{pGBKT7/SfiI}$. The recombinant plasmid wasthenused to transform TOP10 Escherichia coli. Escherichia coli was supplied by American Type Culture Collection and cultured in Luria-Bertani medium $(1 \% \mathrm{NaCl} ; 1 \%$ Polypeptone; $0.5 \%$ Yeast extract) in a humidified incubator at $37^{\circ} \mathrm{C}$ with $5 \% \mathrm{CO}_{2}$. Then, it was inoculated onto Luria-Bertani medium plates $(1 \% \mathrm{NaCl} ; 1 \%$ Polypeptone; $0.5 \%$ Yeast extract; $2 \%$ agar) containing $50 \mu \mathrm{g} / \mathrm{ml}$ kanamycin $\left(\mathrm{LB}-\mathrm{Kan}^{\mathrm{r}+}\right.$ ) overnight at $37^{\circ} \mathrm{C}$. A total of six bacterial colonies were selected and further cultured at $16^{\circ} \mathrm{C}$ overnight with agitation $(250 \mathrm{x} \mathrm{g})$. The plasmids of the cultured bacteria were extracted using a Plasmid Miniprep kit (Qiagen, Inc.) and verified by sequencing.

Agarose gel electrophoresis. Agarose gel electrophoresis is a standard method for identification DNA fragments (15). In the present study, $0.7 \%$ agarose gel was used. Then, $0.14 \mathrm{~g}$ agarose and $20 \mathrm{ml} 1 \mathrm{X}$ Tris-acetate-EDTA buffer in a flask were heated in a microwave for $5 \mathrm{~min}$ at $100^{\circ} \mathrm{C}$. Then, $2 \mu \mathrm{l}$ ethidium bromide was added (Thermo Fisher Scientific, Inc.), and poured onto a taped plate with casting combs. Then, $2 \mu \mathrm{l}$ DNA sample from Escherichia coli was added to the $5 \mathrm{X}$ agarose gel, and underwent electrophoresis at $120 \mathrm{~mA}$ for $40 \mathrm{~min}$ at $25^{\circ} \mathrm{C}$ until separation had been achieved. The DNA fragments were visualized using a long wave UV light box.

Self-activation test.pGADT7, pGBKT7-p53,pGBKT7-laminC and the pGADT7-170297-LUcDNA plasmid genome were provided from Hitech Bio-Technology Co., Ltd. The pGADT7-170297-LUcDNA plasmid genome was referred to as 'pGADT7-largeT'. As a positive control, pGBKT7-p53 and pGADT7-largeT were used to transform AH109 yeast cells. As a negative control, AH109 cells were also transformed using pGBKT7-lamin C with pGADT7-large T. AH109 were purchased from the American Type Culture Collection and cultured in yeast peptone dextrose adenine (YPDA; $1 \%$ Yeast extract; $2 \%$ Tryptone; $2 \%$ Glucose; $0.02 \%$ Adenine) with $5 \% \mathrm{CO}_{2}$. Synthetic defined (SD)/-tryptophan (Trp), SD/-leucine (Leu), SD/-Trp/-Leu, SD/-Trp/-Leu/-histidine (His) and $\mathrm{SD} /$-Trp/-Leu/-His/-adenine (Ade) media were purchased from Clontech Laboratories, Inc.

To test whether the prey plasmid was able to auto-activate the reporter genes, pGADT7 and pGBKT7-CT798 were used to co-transform AH109 cells according to the lithium acetate transformation method (16). The cells were then cultured in SD medium lacking various amino acids (SD/-Trp/-Leu) at $30^{\circ} \mathrm{C}$ for 4 days. The isolated yeast clones were tested for the activation of the third reporter gene, including imidazoleglycerol-phosphate dehydratase (HIS3), phosphoribosylaminoimidazole carboxylase (ADE2) and LacZ.

Yeast two-hybrid screening. The pGADT7-170297-LUcDNA plasmid genome from HeLa cells was used for the yeast 
two-hybrid screen (17). AH109 yeast cells containing the pGBKT7-CT798 plasmid were plated and selected using SD agar plates without tryptophan. The positive clones were inoculated in $50 \mathrm{ml} \mathrm{SD} /$-Trp medium and cultured at $30^{\circ} \mathrm{C}(225 \mathrm{x} \mathrm{g})$ for $18 \mathrm{~h}$. The cells were then transferred to $500 \mathrm{ml}$ YPDA medium and cultured at $30^{\circ} \mathrm{C}$ (agitation at $225 \mathrm{x}$ g) for $4 \mathrm{~h}$ until $\mathrm{OD}_{600}=0.6$. Next, the cultured cells were centrifuged at 4,000 $\mathrm{x}$ g for $5 \mathrm{~min}$ at room temperature; the supernatant was discarded and the pellet was resuspended in $0.1 \mathrm{MLi}$ Ac of $20 \mathrm{ml}$. The cells were then centrifuged once more under the same conditions and suspended in $10 \mathrm{ml}$ LiAc $(0.1 \mathrm{M})$. Then, $9.6 \mathrm{ml}$ 50\% PEG3350, $1.44 \mathrm{ml} \mathrm{LiAc}$, $300 \mu$ single-stranded DNA and $25 \mu \mathrm{g}$ cDNA library plasmid were added, and the cells were cultured at $30^{\circ} \mathrm{C}$ for $30 \mathrm{~min}$. The mixture was heated in a water bath at $42^{\circ} \mathrm{C}$ for $25 \mathrm{~min}$, followed by resuscitation at $30^{\circ} \mathrm{C}$ for $1 \mathrm{~h}$. The cell pellet was resuspended in $8 \mathrm{ml}$ sterile water following centrifugation at $4,000 \mathrm{x}$ g for $5 \mathrm{~min}$ at room temperature. The cells were cultured on SD/-Leu/-Trp/-His and 3-amino-1,2,4-triazole (3AT) plates with $200 \mu \mathrm{l}$ solution/plate, for a total of 40 plates. In order to determine library transformation efficiency, $20 \mu \mathrm{l}$ cell cultures from $8 \mathrm{ml}$ solution were diluted 10 -fold by gradient and cultured on SD/-Leu/-Trp plates. The cells were cultured for $3-4$ days at $30^{\circ} \mathrm{C}$, with observation ofthe conversion results and recording of conversion efficiency. The following calculations were used: Total number of transformants $\left.=\left(n_{1} / 20+n_{2} / 2+n_{3} / 0.2\right) \times 1 / 3 \times 8,000\right)$. Where $n_{1}$, the number of colonies growing on the plate after 10 -fold dilution; $\mathrm{n}_{2}$, the number of colonies growing on the plate after 100 -fold dilution; $\mathrm{n}_{3}$, the number of colonies growing on the plate after 1,000-fold dilution. Transformation efficiency $=$ Total number of transformants $/ 25 \mu \mathrm{g}$ cDNA plasmid.

Positive clone verification. After culturing on $\mathrm{SD} /$-Leu/-Trp/-His and 3AT plates, the positive clones were plated onto SD/-Leu/-Trp plates. To select for clones which potentially interacted with CT798, the clones were inoculated onto SD/-Leu/-Trp/-His+3AT and SD/-Leu/-Trp/-Ade plates to assess the transcriptional activation of the HIS3 and ADE2 reporter genes, and resuspended in sterile water to detect LacZ expression. Concurrently, positive and negative control transformation experiments were conducted, for the purpose of selecting true positive colonies. The positive clone plasmids were subsequently extracted using the Plasmid Miniprep kit. Following sequencing, a BLAST search was conducted using GenBank (National Center for Biotechnology Information) to determine the associated genes.

Rotary validation. The selected clones were used to transform AH109 yeast cells containing pGBKT7-CT798 as aforementioned, and these cells were cultured on $\mathrm{SD} /$-Leu/-Trp plates. Positive clones were transferred onto $\mathrm{SD} /$-Leu/-Trp/-His/-Ade+3AT and SD/-Leu/-Trp/-Ade plates to determine the transcriptional activation of the HIS3 and ADE2 reporter genes, and resuspended in sterile water to detect LacZ expression.

Co-immunoprecipitation. HeLa cells were provided from the University of South of China and cultured in complete DMEM (Gibico; Thermo Fisher Scientific, Inc), containing
10\% FBS (Gibico; Thermo Fisher Scientific, Inc.) in a humidified incubator at $37^{\circ} \mathrm{C}$ with $5 \% \mathrm{CO}_{2}$. HeLa cells were infected with $C$. trachomatis. $C$. trachomatis was added into HeLa cell. At $48 \mathrm{~h}$ post-infection, the cells were washed twice with cold PBS, and lysed with RIPA buffer (Epizyme, Inc.) for $15 \mathrm{~min}$ at $4{ }^{\circ} \mathrm{C}$. The lysate was pre-clarified by rotation for $10 \mathrm{~min}$, centrifuged at $14,000 \mathrm{x}$ g for $15 \mathrm{~min}$ (both $4^{\circ} \mathrm{C}$ ), and incubated with protein-A agarose beads. Cell-free lysates were incubated with a rabbit anti-PHB antibody (1:1,000; Cell Signaling Technology, Inc.; cat. no. ab75766.) or IgM antibody (1:1,000; Cell Signaling Technology, Inc.) at $4^{\circ} \mathrm{C}$ overnight. Protein-A beads were added for $2 \mathrm{~h}$ at room temperature, and the lysates were subsequently washed three timeswith PBS. The proteins were eluted into 5X SDS-PAGE sample buffer, separated by $12 \%$ SDS-PAGE gel for immunoblotting.

Western blotting. HeLa cells were infected with C. trachomatis and harvested at $48 \mathrm{~h}$ post-infection. Cells were washed with cold PBS and lysed with RIPA buffer (Epizyme, Inc.) with protease inhibitor and phosphatase inhibitor. Then, $10 \mu \mathrm{l}$ protein extracts and cell lysates (Input control) were separated by $12 \%$ SDS-PAGE gel and electrophoretically transferred to nitrocellulose membranes. After blocking in 5\% non-fat dry milk with TBST $(50 \mathrm{mM}$ Tris- $\mathrm{HCl} ; \mathrm{pH} 7.4 ; 5.36 \mathrm{mM} \mathrm{KCl}$; $274 \mathrm{mM} \mathrm{NaCl}$; $0.1 \%$ Tween-20) for $2 \mathrm{~h}$ at room temperature, the membranes were probed with a mouse anti-CT798 antibody (1:1,000; The University of South China) overnight at $4^{\circ} \mathrm{C}$. Then, the membrane waswashed four times for $5 \mathrm{~min}$ in TBST and incubated with anti-mouse horseradish peroxidase-conjugated secondary antibody (1:3,000; Servicebio, Inc.; cat. no. GB23303) for $1 \mathrm{~h}$ at $37^{\circ} \mathrm{C}$ Blots were detected by Western Blot system G: BOX Chemi XXX9 (Syngene International Ltd.).

\section{Results}

Construction of the pGBKT7-CT798 bait plasmid. CT798 is $53 \mathrm{kDa}$; the full length of CT798 (1,440 bp) was successfully amplified from C. trachomatis genomic DNA viaPCR, and analyzed using restriction enzyme digestion (Fig. 1A). The PCR products were subsequently cloned into the bait vector pGBKT7. The construct was transformed into E. coli and inoculated onto LB-kan ${ }^{\mathrm{r}+}$ plates. Following incubation, six positive transformants were randomly picked and identified by PCR (Fig. 1B), and the results of recombinant plasmid sequencing were consistent with that of pGBKT7-CT798.

Validity of the bait plasmid pGBKT7-CT798. The AH109 yeast strain contains various nutritional reporter genes. These include: i) ADE2, coding for phosphoribosylaminoimidazole carboxylase, an important enzyme for adenine synthesis; ii) HIS3, encoding imidazoleglycerol-phosphate dehydratase, another key enzyme for adenine synthesis; and iii) LacZ, which encodes $\beta$-galactosidase enzymes. Therefore, AHI09 allows for the control of strong nutritional selection, whilst reducing the incidence of false positives. To determine the efficiency of the system, pGBKT7-CT798 and pGADT7 were used to transform AH109 cells, and pGBKT7-p53 and pGBKT7-laminC were used as the positive 


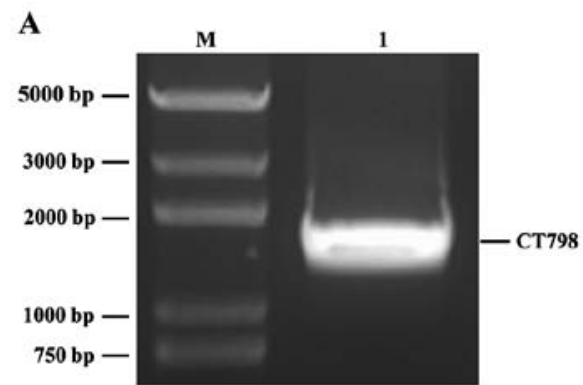

B

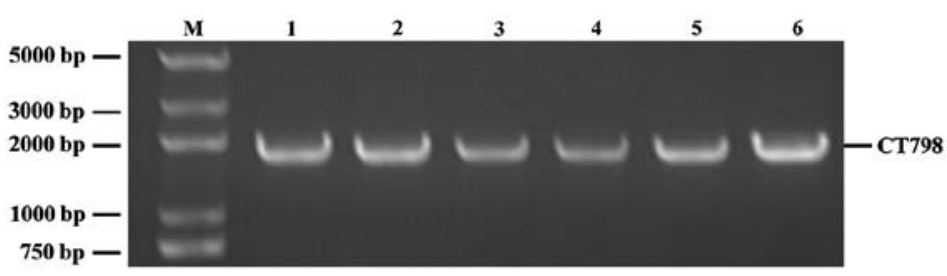

Figure 1. Construction of the bait plasmid. PCR products were separated on a $1 \%$ agarose gel, and the DNA was visualized using ethidium bromide staining. (A) Restriction enzyme digestion of pET28a-CT798. (B) CT798PCR product from different clones. CT798, Chlamydia trachomatis glycogen synthase (also known as $\mathrm{Glg} \mathrm{A}$ ).

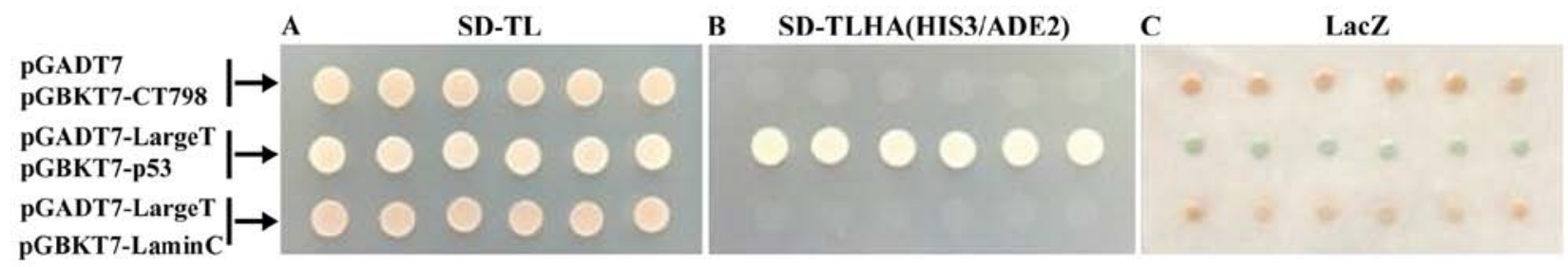

Figure 2. CT798 has no self-activation function. Growth of AH109 cells containing pGADT7/pGBKT7-CT798 and pGADKT7-Large/pGBKT7-P53 on plates with different nutrient deficiencies. pGADKT7-Large/pGBKT7-P53 was used as the positive control, and pGADT7-LargeT/pGBKT7-LaminC as the negative control. (A) Diploid growth on SD-TL plates. Only the positive control can (B) be grown on SD-TLHA plates and (C) express LacZ. CT798, Chlamydia trachomatis glycogen synthase (also known as GlgA); SD, synthetic defined; -TLHA, -tryptophan/-leucine/-histidine/-adenine; HIS3, imidazoleglycerol-phosphate dehydratase; ADE2, phosphoribosylaminoimidazole carboxylase.

A

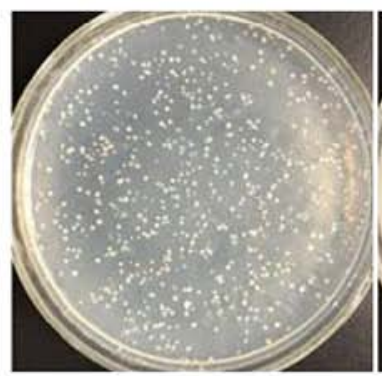

$1 / 10 \quad n=808$

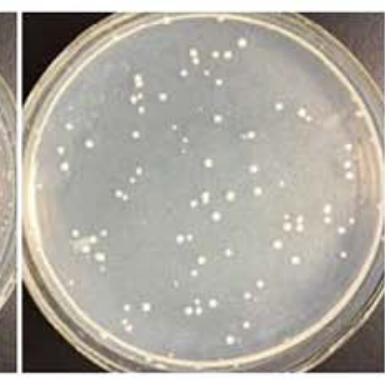

$1 / 100 n=103$

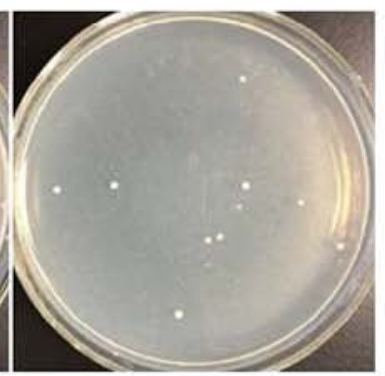

$1 / 1000 n=10$
B

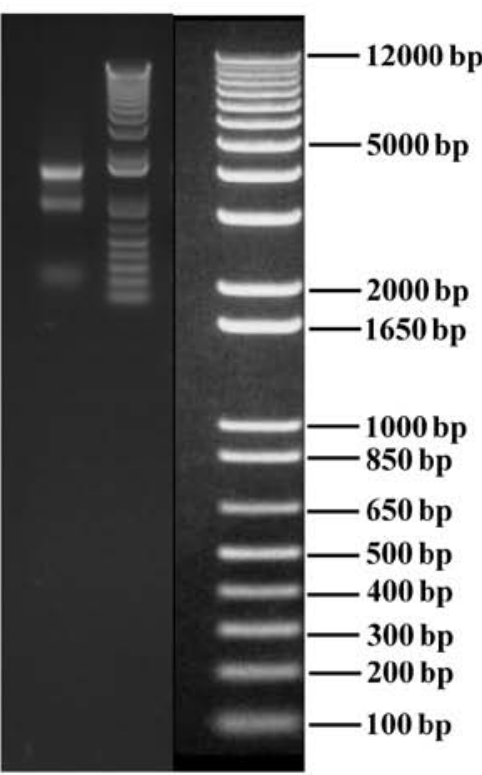

Figure 3. Transformation efficiency. (A) A total of $25 \mu \mathrm{g}$ cDNA library plasmid was used to transform AH109 yeast cells containing pGBKT7-CT798. The cell pellet was resuspended in $8 \mathrm{ml}$ sterile water, serially diluted, and $20 \mu \mathrm{l}$ was cultured on SD/-leucine/-tryptophan plates. Total number of transformants $=(808 / 20)+(103 / 2)+(10 / 0.2) \times 1 / 3 \times 8,000=3.78 \times 10^{5}$. Transformation efficiency $=3.78 \times 10^{5} / 25 \mu \mathrm{g}=1.51 \times 10^{4} / \mu \mathrm{g}$. (B) Agarose gel electrophoresis of different clones.

and negative controls, respectively. AH109 cells were then cultured on SD/-Leu/-Trp plates, and six transformants were used to test for self-activation, including pointing-plate culturing on SD/-Leu/-Trp/-His/-Ade plates to test for the expression of HIS3 and ADE2, and a colorimetric assay to determine the expression of LacZ. Control strains both grow on the SD/-Leu/-Trp plate, but only the positive control cells are able to grow on the SD/-Leu/-Trp/-His/-Ade plate. In the present study, AH109 cells containing the pGBKT7/CT798 plasmid grew on the SD/-Leu/-Trp plate, but not on the $\mathrm{SD} /$-Leu/-Trp/-His/-Ade plate. Furthermore, the results of the colorimetric assay were consistent with those of the negative control (Fig. 2). These results suggest that CT798 does not possess self-activation function in AH109 cells. 

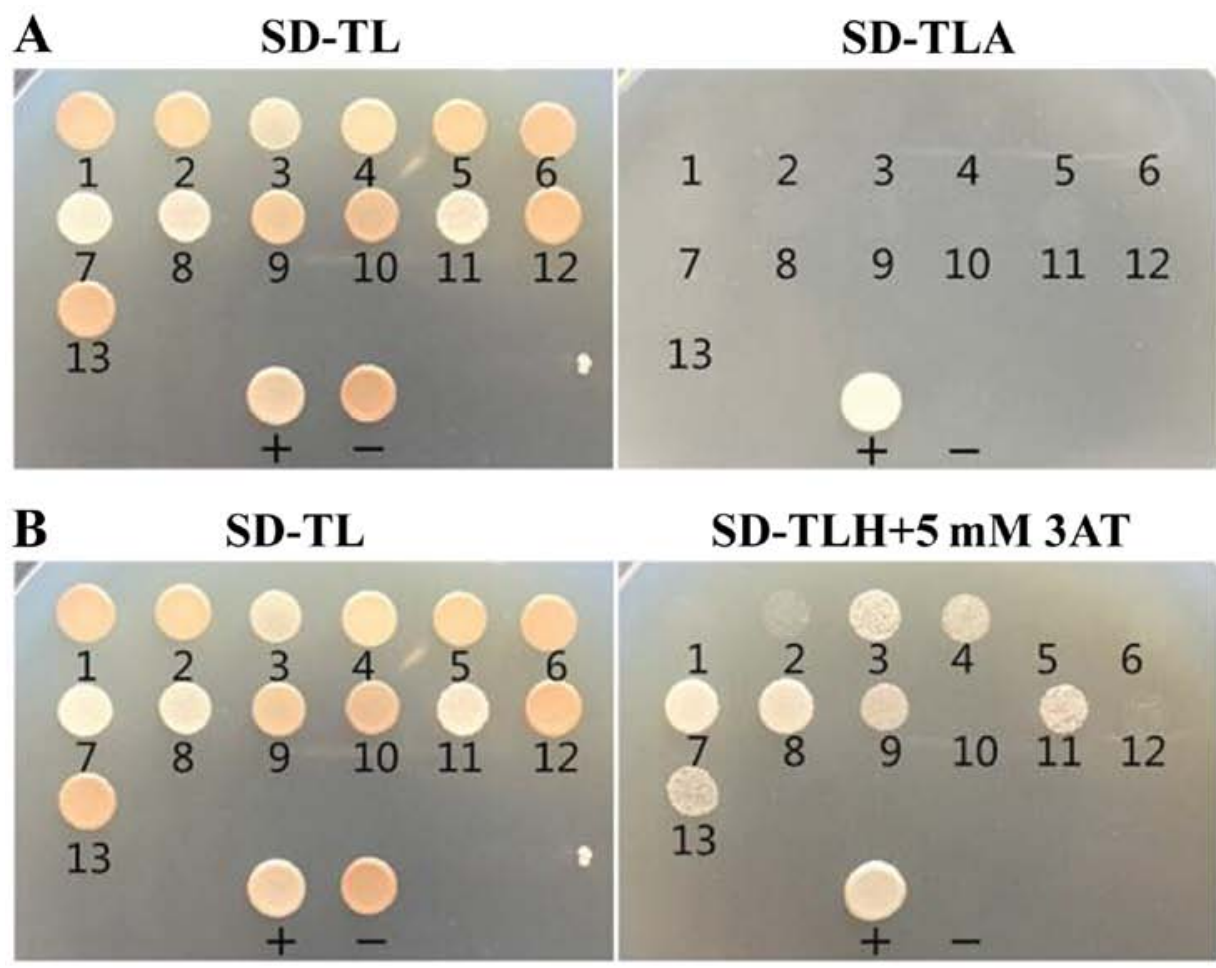

SD-TLH+5 mM 3AT
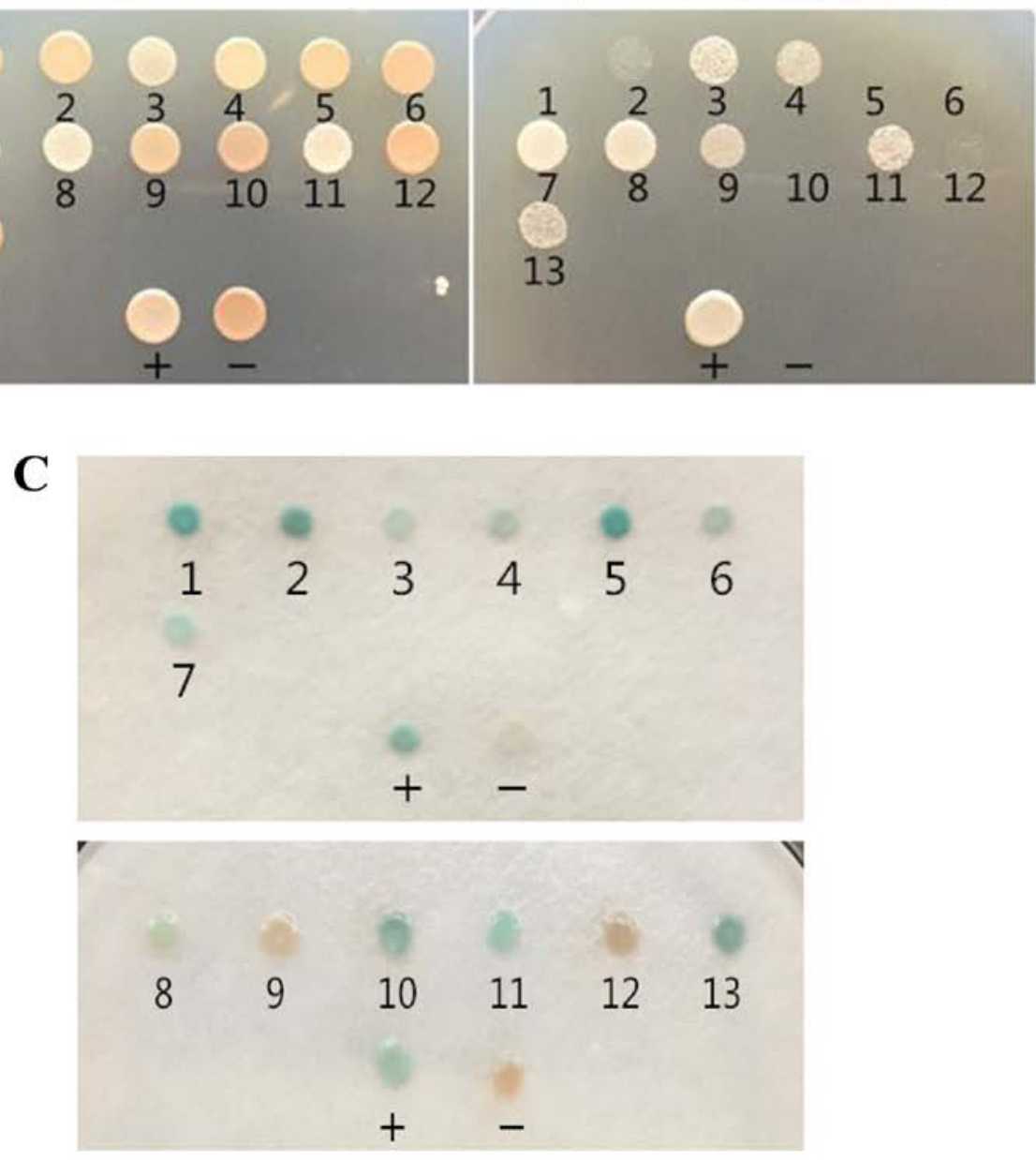

Figure 4. Yeast two-hybrid screening. Interactions identified by yeast-two hybrid analysis were verified by screening diploids grown on SD-TL plates, and in high stringency medium. (A) No interactions were detected on SD-TLA plates, resulting in blue yeast colonies. (B) A total of seven interactions were identified using SD-LTH +3AT plates, and (C) 11 interactions activated the LacZ reporter gene. SD, synthetic defined; -TLHA, -tryptophan/-leucine/-histidine/-adenine; 3AT, 3-amino-1,2,4-triazole.

cDNA library screening. pGADT7-170297-LUcDNA library used for screening was supplied by Hitech Bio-Technology Co., Ltd. Plasmids contained only pGBKT7-CT798 as bait for screening the pGADT7-170297-LUcDNA library. Library plasmids were transformed into AH109 cells containing bait plasmid and cultured on SD/-Leu/-Trp/-His and 3AT plates. AH109 cells were also cultured on SD/-Leu/-Trp plates (Fig. 3), and transformation efficiency (which is important for the success of the yeast two-hybrid system) was determined. The results revealed a total of $3.78 \times 10^{5}$ transformants, indicating a transformation efficiency of $1.51 \times 10^{4} / \mu \mathrm{g}$. At the same time, the quality of the yeast two-hybrid library was assessed. Agarose gel electrophoresis of the PCR products indicated the average length of the insertion product to be $\sim 1.5 \mathrm{~kb}$, and the positive success rate of the library was $100 \%$.

Identification of proteins interacting with the bait protein. In the present study, 13 positive clones were obtained from two rounds of selection, which were designated A1-13. These clones were not capable of growing on SD/-Leu/-Trp/-Ade plates, and seven grew on SD/-Leu/-Trp/-His and 3AT plates (Fig. 4). In addition, 11 of the 13 clones expressed LacZ. 
Table I. Biological information of the seven positive clones.

\begin{tabular}{|c|c|c|}
\hline Clone(s) & NCBI accession & NCBI description \\
\hline A3 & XM_017025718.1 & $\begin{array}{l}\text { PREDICTED: Homo sapiens } \mathrm{CXXC} \text { finger protein } 1 \text {, transcript variant } \\
\mathrm{X} 2 \text {, mRNA }\end{array}$ \\
\hline A4, A9 & XM_017024763.1 & PREDICTED: Homo sapiens prohibitin, transcript variant $\mathrm{X} 2$, mRNA \\
\hline $\mathrm{A} 7, \mathrm{~A} 8$ & NM_001256139.1 & $\begin{array}{l}\text { Homo sapiens capping actin protein, gelsolin-like, transcript variant } 2 \text {, } \\
\text { mRNA }\end{array}$ \\
\hline $\mathrm{A} 11, \mathrm{~A} 13$ & AK298222.1 & $\begin{array}{l}\text { Homo sapiens cDNA FLJ56357 complete cds, highly similar to } \\
\text { Homo sapiens apolipoprotein A-I binding protein, mRNA }\end{array}$ \\
\hline
\end{tabular}

NCBI, National Center for Biotechnology Information.

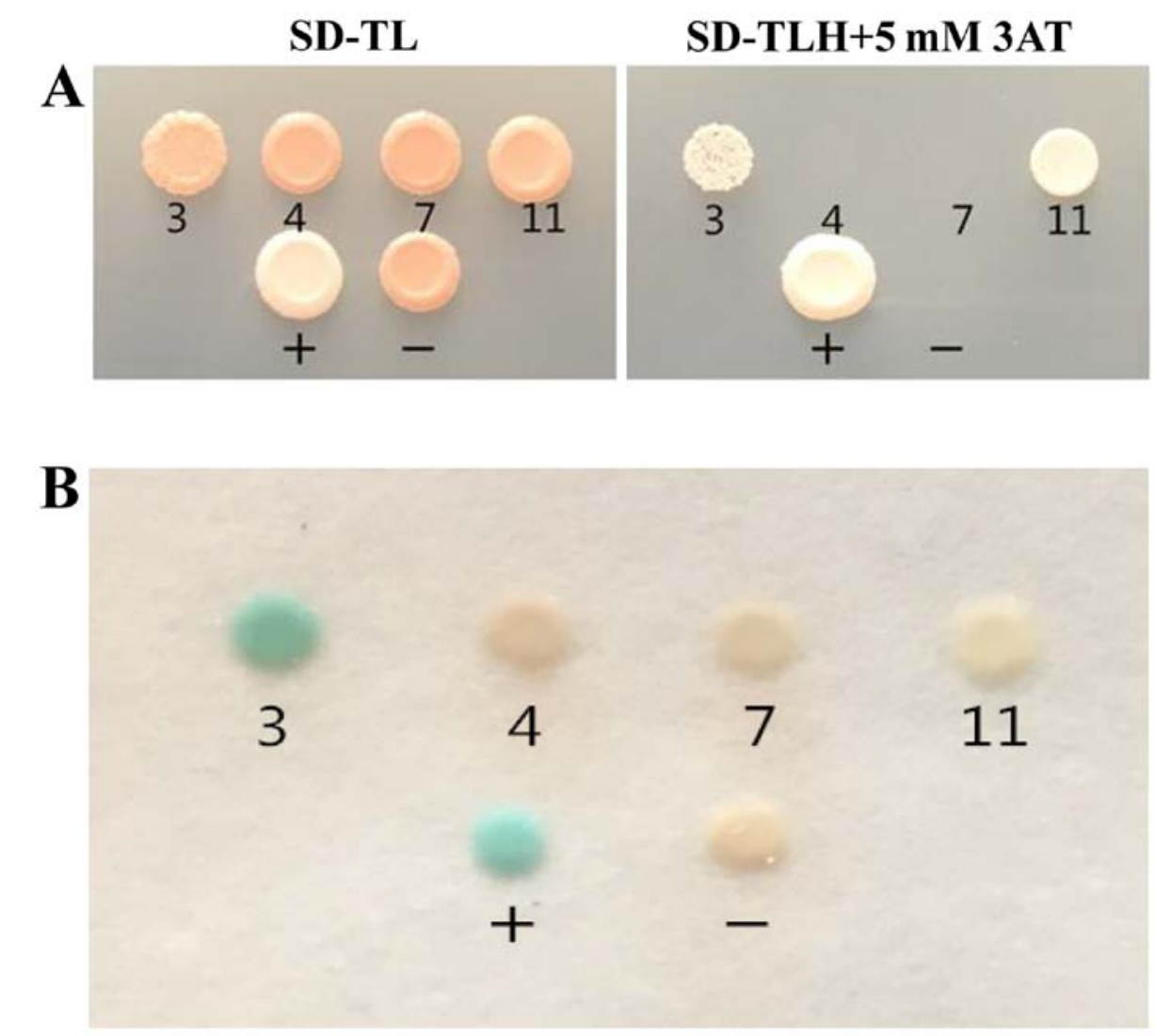

Figure 5. Rotary validation. After screening, clones for the four selected genes were used to transform into AH109 yeast cells containing pGBKT7-CT798. The diploids were cultured on SD-TL plates and in high stringency medium. (A) Clones A3 and A11 successfully grew on SD-TLH +3AT plates, and (B) clone A3 was able to active the LacZ gene. SD, synthetic defined; -TLH, -tryptophan/-leucine/-histidine; 3AT, 3-amino-1,2,4-triazole.

These results indicated that the HIS3 gene was activated by the seven positive clones, and further suggested that A3, A4, A7, A8, A9, A11 and A13 interacted with CT798 to a certain degree. The positive clones were subsequently sequenced and analyzed using BLAST software. The results indicated that the seven positive clones possessed a high degree of similarity to known genes encoding four different proteins (Table I). Finally, for rotary validation, the resulting four plasmids were used to transform AH109 cells containing the pGBKT7-CT798 plasmid (Fig. 5). The results revealed that clones A3 and A11 were able to active the HIS3 gene, and that only A3 activated the LacZ gene.
PHB interacts with CT798. Co-immunoprecipitation was performed to validate the interaction between CT798 and PHB (Fig. 6). CT798 was co-precipitated by anti-PHB from the lysates of cells infected with $C$. trachomatis, but not by precipitation with IgM. This suggested that PHB interacts with CT798.

\section{Discussion}

GlgA is a key enzyme involved in glycogen metabolism, which catalyzes the glucose moiety of UDP-glucose transferred to the non-reducing end of a linear $\alpha-1,4$-glucan primer. Previous 


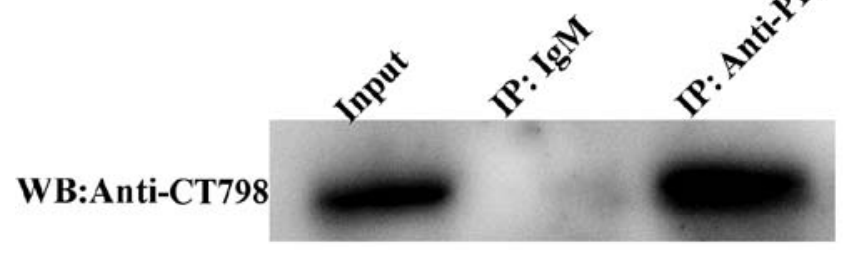

Figure 6. Interaction between CT798 and PHB. HeLa cells were infected with $C$. trachomatis, harvested $48 \mathrm{~h}$ post-infection, and the lysates were prepared for co-immunoprecipitation. Supernatants from the infected lysates were incubated with protein-A beads that had been pre-loaded with anti-PHB or the negative control antibody IgM. Immunoblots were probed with anti-CT798. CT798, Chlamydia trachomatis glycogen synthase; PHB, prohibitin; WB, western blotting.

studies have reported that the inactivation of GlgA not only inhibits glycogen synthesis, but also reduces bacterial stress resistance $(18,19)$. It is possible that $\mathrm{Glg} \mathrm{A}$ is associated with adaptive environmental survival, colonization and virulence. In addition, the average chlamydial genome size is $\sim 1.0 \mathrm{Mb}$, only $1 / 4$ of that of E. coli (20). Notably, the expression and secretion of GlgA is highly conserved among different serovars of $C$. trachomatis and C. muridarum, a separate, but related species (21). The importance of GlgA in chlamydial growth and development is well established (10). However, the effects of GlgA secretion into the host cell and its involvement in chlamydial metabolism (which may serve a role in the association of GlgA secretion with the inflammatory response) are yet to be investigated. Thus, screening for binding partners of GlgA using the yeast two-hybrid system will enhance current understanding of the role of GlgA in chlamydial biology and pathogenesis.

Yeast two-hybrid technology, an effective means of detecting unidentified protein-protein interactions, has allowed for further exploration of biological pathways and their regulation (22). In the present study, not only were positive and negative controls used, but promoters modulating HIS3, ADE2 and LacZ reporter gene expression in the AH109 yeast strain were also included to exclude false-positive results. Although the yeast two-hybrid assay is associated with high sensitivity and easy operation, it exhibits obvious drawbacks, and false positives are still easily obtained. Hence, other in vitro assays (such as immunoprecipitation) should be conducted to verify these results and improve data credibility.

To enhance the probability of identifying unambiguous interactions, stringent media were used, which lacked the amino acid histidine or the nucleic acid adenine (23). Growth-dependent selection enables the most effective identification of interaction partners. Therefore, only bait-prey interactions result in HIS3 or ADE2 reporter gene expression; LacZ expression provides a reference to reflect the strength of the interactions. The identified interaction proteins were Homo sapiens CXXC finger protein 1 (CFP1), PHB, gelsolin-like actin-capping protein (CAPG) and apolipoprotein A-I binding protein (ApoA-I).

CFP1, an unmethylated $\mathrm{CpG}$-binding protein, is a component of the mammalian setl histone methyltransferase complex, which is involved in histone methylation, regulating T-cell development and promoting cellular differentiation (24). Anti-chlamydial $\mathrm{T}$ cells may represent a double-edged sword, and may be responsible for initiating the pathological changes associated with chlamydial infection (25). Thus, T-cell secretion of GlgA and CFP1 may promote chlamydial persistence and immunopathology.

PHB is characterized as an anti-proliferative gene, and is widely distributed in different cellular compartments, such as the plasma membrane, nucleus and mitochondria (26). PHB family members possess two subunits, PHB1 and PHB2; PHB1 binds to the transcription factor p53, inducing p53-inducible gene 3 promoter binding and promoting p53-dependent cell apoptosis (27). PHB2 can translocate from the mitochondria to the nucleus during capsaicin-induced apoptosis (28). PHB1 and 2 are conserved mitochondrial proteins with diverse functions, which include roles in cell proliferation and mitochondrial integrity (29). As obligate intracellular pathogens, chlamydia have been proposed to be anti-apoptotic to complete their development cycle (30). Accordingly, PHB is hypothesized to shuttle between the mitochondria and the nucleus during the cell cycle, and GlgA is secreted into the cytosol at $\sim 24 \mathrm{~h}$ post-infection (31). These findings indicate that at the beginning of the developmental cycle, the manipulation of GlgA by C. trachomatis may prevent premature release of inclusion bodies from the cell via its interaction with PHB.

The actin-binding protein CAPG was originally identified in the cytoplasm of alveolar macrophages (32). CAPG interacts with cytoplasmic and nuclear proteins that are closely associated with cell proliferation and motility (33). It promotes restructuring of the actin cytoskeleton by capping the fast-growing end of actin filaments in a $\mathrm{Ca}^{2+}$ and phosphatidylinositol 4,5-bisphosphate-dependent manner, but does not initiate actin filament severing (34). Furthermore, CAPG may be involved in mediating cross-talk between the actin cytoskeleton and the microtubule-based organelles that regulate cell division (35). C. trachomatis has evolved exclusion mechanisms of host-cell escape, through which membrane-bound inclusions are released from the host cell viaa process resembling exocytosis (36). This process is hypothesized to be dependent on cytoskeletal activities, including actin polymerization, as well as neural Wiskott-Aldrich syndrome protein, Rho GTPase and the myosin motor complex (37). The results of the present study demonstrated that CAPG may interact with GlgA to regulate extrusion formation, which facilitates evasion of the localized inflammatory response at the site of infection.

ApoA-I is the primary constituent of plasma high-density lipoprotein (HDL) and plays a central role in reverse cholesterol transport (RCT) (38). The process of RCT, which transports cellular cholesterol back to the liver, is considered to be one of the most important anti-atherogenic properties of HDL (39). Several experimental strategies aimed at overexpressing ApoA-I are associated with the anti-atherogenic effects of HDL (40). Besides upper respiratory infection, chlamydial challenge has also been associated with chronic inflammatory conditions, including atherosclerosis (41). The interaction between GlgA and ApoA-I has been speculated as a novel target for the treatment and prevention of severe long-term sequelae of chlamydial infections. As these interacting proteins are known to be associated with immunoregulation, apoptosis and tumor progression, it is possible that GlgA operates in a similar manner.

In the present study, four potential GlgA-interaction proteins were identified. However, three plasmids did not 
pass rotary validation, which may be due to unsuccessful transformation of prey or bait plasmids into AH109 or weak protein-protein interactions. Here, full-length of PHB was found to interact with GlgA via co-immunoprecipitation. But unfortunately, the part of PHB that interacts with GlgA was not determined in this study, even though PHB1 and PHB2 belong to the PHB family. Thus, subsequent studies will investigate the interacting part of GlgA to further clarify the relation between $C$.trachomatis infections and GlgA secretion into the host cell cytosol. In summary, the yeast two-hybrid system was successfully exploited to screen for GlgA interaction partners, indicating further regulatory functions of $\mathrm{Glg} \mathrm{A}$.

\section{Acknowledgements}

Not applicable.

\section{Funding}

This project was supported by the Natural Science Foundation of China (grant nos. 81471969, 81202374 and 31600150), the construct program of Hunan Provincial Key Laboratory for Special Pathogens Prevention and Control Foundation (grant no. 2014-5), and Hunan Province Cooperative Innovation Center for Molecular Target New Drug Study (grant no. 2015-351).

\section{Availability of data and materials}

All data generated or analyzed during this study are included in this published article.

\section{Authors' contributions}

ZS, YS, YL, XL, HC, HW and BP performed the experiments. $\mathrm{ZS}$ wrote the paper. CL designed the study, coordinated the data analysis, reviewed and edited the manuscript. All authors read and approved the manuscript.

\section{Ethics approval and consent to participate}

Not applicable.

\section{Patient consent for publication}

Not applicable.

\section{Competing interests}

The authors declare that they have no competing interests.

\section{References}

1. Wang Y, Cutcliffe LT, Skilton RJ, Ramsey KH, Thomson NR and Clarke IN: The genetic basis of plasmid tropism between Chlamydia trachomatis and Chlamydia muridarum. Pathog Dis 72: 19-23, 2014.

2. Weber MM, Lam JL, Dooley CA, Noriea NF, Hansen BT, Hoyt FH, Carmody AB, Sturdevant GL and Hackstadt T: Absence of specific Chlamydia trachomatis inclusion membrane proteins triggers premature inclusion membrane lysis and host cell death. Cell Rep 19: 1406-1417, 2017.
3. Whitcher JP, Srinivasan M and Upadhyay MP: Corneal blindness: A global perspective. Bull World Health Organ 79: 214-221, 2001.

4. Brunham RC and Rey-Ladino J: Immunology of Chlamydia infection: Implications for a Chlamydia trachomatis vaccine. Nat Rev Immunol 5: 149-161, 2005.

5. Yang C, Starr T, Song L, Carlson JH, Sturdevant GL, Beare PA, Whitmire WM and Caldwell HD: Chlamydial lytic exit from host cells is plasmid regulated. mBio 6: e01648-15, 2015.

6. Moulder JW: Interaction of chlamydiae and host cells in vitro. Microbiol Rev 55: 143-190, 1991.

7. Stephens RS, Myers G, Eppinger M and Bavoil PM: Divergence without difference: Phylogenetics and taxonomy of Chlamydia resolved. FEMS Immunol Med Microbiol 55: 115-119, 2009.

8. Zhong G: Chlamydial plasmid-dependent pathogenicity. Trends Microbiol 25: 141-152, 2017.

9. Patton MJ, McCorrister S, Grant C, Westmacott G, Fariss R, Hu P, Zhao K, Blake M, Whitmire B, Yang C, et al: Chlamydial protease-like activity factor and type III secreted effectors cooperate in inhibition of p65 nuclear translocation. mBio 7: pii: e01427-16, 2016.

10. Lu C, Lei L, Peng B, Tang L, Ding H, Gong S, Li Z, Wu Y and Zhong G: Chlamydia trachomatis GlgA is secreted into host cell cytoplasm. PLoS One 8: e68764, 2013.

11. O'Connell CM, Ingalls RR, Andrews CW Jr, Scurlock AM and Darville T: Plasmid-deficient Chlamydia muridarum fail to induce immune pathology and protect against oviduct disease. J Immunol 179: 4027-4034, 2007.

12. Ma RR, Sun J, Fang WH, Dong YP, Ruan JM, Yang XL and $\mathrm{Hu} \mathrm{K}$ : Identification of Carassius auratus gibelio liver cell proteins interacting with the $\mathrm{GABA}_{\mathrm{A}}$ receptor $\gamma 2$ subunit using a yeast two-hybrid system. Fish Physiol Biochem 45: 199-208, 2019.

13. Fields S and Song O: A novel genetic system to detect protein-protein interactions. Nature 340: 245-246, 1989.

14. Nguyen PH, Lutter EI and Hackstadt T: Chlamydia trachomatis inclusion membrane protein MrcA interacts with the inositol 1,4,5-trisphosphate receptor type 3 (ITPR3) to regulate extrusion formation. PLoS Pathog 14: e1006911, 2018.

15. Lee PY, Costumbrado J, Hsu CY and Kim YH: Agarose gel electrophoresis for the separation of DNA fragments. J Vis Exp: pii: 3923, 2012.

16. Gietz RD and Schiestl RH: High-efficiency yeast transformation using the LiAc/SS carrier DNA/PEG method. Nat Protoc 2: 31-34, 2007.

17. Zhao X, Li P, An K, Jia X, Cheng Y and Jia T: Chlamydia pneumoniae inclusion membrane protein Cpn0147 interacts with host protein CREB3. PLoS One 12: e0185593, 2017.

18. Velmurugan R and Incharoensakdi A: Disruption of polyhydroxybutyrate synthesis redirects carbon flow towards glycogen synthesis in synechocystis sp. PCC 6803 overexpressing glgC/glgA. Plant Cell Physiol 59: 2020-2029, 2018.

19. Preiss J: Bacterial glycogen synthesis and its regulation. Annu Rev Microbiol 38: 419-458, 1984.

20. Stephens RS, Kalman S, Lammel C, Fan J, Marathe R, Aravind L, Mitchell W, Olinger L, Tatusov RL, Zhao Q, et al: Genome sequence of an obligate intracellular pathogen of humans: Chlamydia trachomatis. Science 282: 754-759, 1998.

21. Everett KD, Bush RM and Andersen AA: Emended description of the order Chlamydiales, proposal of Parachlamydiaceae fam. nov. and Simkaniaceae fam. nov., each containing one monotypic genus, revised taxonomy of the family Chlamydiaceae, including a new genus and five new species, and standards for the identification of organisms. Int J Syst Bacteriol 49: 415-440, 1999.

22. Stynen B, Tournu H, Tavernier J and Van Dijck P: Diversity in genetic in vivo methods for protein-protein interaction studies: From the yeast two-hybrid system to the mammalian split-luciferase system. Microbiol Mol Biol Rev 76: 331-382, 2012.

23. Peterson TA, Stamnes MA and Piper RC: A yeast 2-hybrid screen in batch to compare protein interactions. J Vis Exp, 2018.

24. Cao W, Guo J, Wen X, Miao L, Lin F, Xu G, Ma R, Yin S, Hui Z, Chen T, et al: CXXC finger protein 1 is critical for T-cell intrathymic development through regulating $\mathrm{H} 3 \mathrm{~K} 4$ trimethylation. Nat Commun 7: 11687, 2016.

25. Wizel B, Nystrom-Asklin J, Cortes C and Tvinnereim A: Role of CD8(+)T cells in the host response to Chlamydia. Microbes Infect 10: 1420-1430, 2008.

26. Yang J, Li B and He QY: Significance of prohibitin domain family in tumorigenesis and its implication in cancer diagnosis and treatment. Cell Death Dis 9: 580, 2018. 
27. Guan X, Liu Z, Wang L, Johnson DG and Wei Q: Identification of prohibitin and prohibiton as novel factors binding to the p53 induced gene 3 (PIG3) promoter (TGYCC)(15) motif. Biochem Biophys Res Commun 443: 1239-1244, 2014.

28. Kuramori C, Azuma M, Kume K, Kaneko Y, Inoue A Yamaguchi Y, Kabe Y, Hosoya T, Kizaki M, Suematsu M and Handa H: Capsaicin binds to prohibitin 2 and displaces it from the mitochondria to the nucleus. Biochem Biophys Res Commun 379: 519-525, 2009.

29. Signorile A, Sgaramella G, Bellomo F and De Rasmo D: Prohibitins: A critical role in mitochondrial functions and implication in diseases. Cells 8: pii: E71, 2019.

30. Fischer SF, Vier J, Kirschnek S, Klos A, Hess S, Ying S and Häcker G: Chlamydia inhibit host cell apoptosis by degradation of proapoptotic BH3-only proteins. J Exp Med 200: 905-916, 2004.

31. Yu Y: Prohibitin shuttles between mitochondria and the nucleus to control genome stability during the cell cycle. Plant Physiol 179: $1435-1436,2019$

32. Yu FX, Johnston PA, Südhof TC and Yin HL: gCap39, a calcium ion- and polyphosphoinositide-regulated actin capping protein. Science 250: 1413-1415, 1990.

33. Li T, Hong X, Zhao J, Teng Y, Zheng J, Chen $\mathrm{H}$, Chen $\mathrm{H}$ and Li H: Gelsolin-like actin-capping protein is associated with patient prognosis, cellular apoptosis and proliferation in prostate cancer. Biomark Med 10: 1251-1260, 2016.

34. Papala A, Sylvester M, Dyballa-Rukes N, Metzger S and D'Haese J: Isolation and characterization of human CapG expressed and post-translationally modified in Pichia pastoris. Protein Expr Purif 134: 25-37, 2017.
35. Hubert T, Van Impe K, Vandekerckhove $\mathrm{J}$ and Gettemans J: The actin-capping protein CapG localizes to microtubule-dependent organelles during the cell cycle. Biochem Biophys Res Commun 380: 166-170, 2009 .

36. Hybiske K and Stephens RS: Mechanisms of host cell exit by the intracellular bacterium Chlamydia. Proc Natl Acad Sci USA 104: 11430-11435, 2007.

37. Lutter EI, Barger AC, Nair V and Hackstadt T: Chlamydia trachomatis inclusion membrane protein CT228 recruits elements of the myosin phosphatase pathway to regulate release mechanisms. Cell Rep 3: 1921-1931, 2013.

38. White CR, Datta G, Wilson L, Palgunachari MN and Anantharamaiah GM: The apoA-I mimetic peptide 4F protects apolipoprotein A-I from oxidative damage. Chem Phys Lipids 219: 28-35, 2019.

39. Kingwell BA, Chapman MJ, Kontush A and Miller NE: HDL-targeted therapies: Progress, failures and future. Nat Rev Drug Discov 13: 445-464, 2014.

40. Rye KA and Barter PJ: Cardioprotective functions of HDLs. J Lipid Res 55: 168-179, 2014.

41. Campbell LA and Kuo CC: Chlamydia pneumoniae-an infectious risk factor for atherosclerosis? Nat Rev Microbiol 2: 23-32, 2004.

This work is licensed under a Creative Commons Attribution-NonCommercial-NoDerivatives 4.0 International (CC BY-NC-ND 4.0) License. 